\title{
OTEC Thermal Resource Report for Ivory Coast
}

May 1979

Prepared for

U.S. Department of Energy

Assistant Secretary for Energy Technology

Division of Central Solar Technology

Under Contract No. ET-78-C-01-2898 


\section{DISCLAIMER}

This report was prepared as an account of work sponsored by an agency of the United States Government. Neither the United States Government nor any agency Thereof, nor any of their employees, makes any warranty, express or implied, or assumes any legal liability or responsibility for the accuracy, completeness, or usefulness of any information, apparatus, product, or process disclosed, or represents that its use would not infringe privately owned rights. Reference herein to any specific commercial product, process, or service by trade name, trademark, manufacturer, or otherwise does not necessarily constitute or imply its endorsement, recommendation, or favoring by the United States Government or any agency thereof. The views and opinions of authors expressed herein do not necessarily state or reflect those of the United States Government or any agency thereof. 


\section{DISCLAIMER}

Portions of this document may be illegible in electronic image products. Images are produced from the best available original document. 
Available from:

National Technical Information Service (NTIS)

U.S. Department of Commerce

5285 Port Royal Road

Springfie1d, Virginia 22161

Price: Printed copy: $\$ 4.50$

Microfiche: $\$ 3.00$ 
HCP/T2898-01/1

Dist Cat UC-

\section{OTEC Thermal Resource Report for Ivory Coast}

May 1979

Prepared for

\section{U.S. Department of Energy}

Assistant Secretary for Energy Technology

Division of Central Solar Technology

Washington, D.C. 20585

Prepared by

William Alan Wolff

Ocean Data Systems, Inc.

Monterey, California 93940

Under Contract No. ET-78-C-01-2898

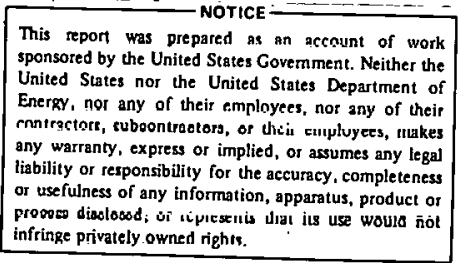




\section{NOTICE}

This report was prepared as an accoint of work sponsored by the United States Government. Neither the United States nor the United States Department of Energy. nor any of their employees, makes any warranty, express or implied, or assumes any legal liability or responsibility for the accuracy, completeness, or usefulness of any intormation, apparatus, product, or process disclosed, or represents

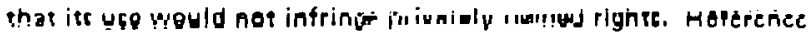
herain to any specific commercial producr, procèss, or service by trade name, mark, manufacturer, or otherwise, does not necessarily constitute or imply its endorsement, recommendation, or favoring by the United States Government or any agency thereof. The views and opinions of authors expressed herein do not necessarily state or reflect those of the United States Government or any agency thereof. 
INTRODUCTION $\ldots \ldots \ldots \ldots \ldots \ldots \ldots \ldots \ldots \ldots \ldots \ldots \ldots \ldots \ldots \ldots \ldots \ldots$

I.

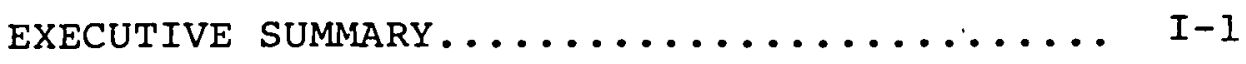

II. BATHYMETRY .................... II-1

III. THERMAL RESOURCE. III-I

IV. MIXED LAYER DEPTH. IV -1

V. . WEATHER CONDITIONS. $\mathrm{V}-1$

VI. SEA AND SWELL CHARACTERISTICS. VI-1

VII. CURRENTS. 
INTRODUCTION

One of the basic environmental considerations in site selection for an Ocean Thermal Energy Conversion (OTEC) power plant is the availability of an adequate temperature difference resource. OTEC plants are designed to convert the potential energy in the temperature difference between the warm ocean surface water and the cold water existing at deeper depths into electricity. The turbines which produce the electricity must run on temperature differentials which are extremely small by the standards of conventional energy plants. Therefore, a definition of the most probable temperature structure for a site is most important.

In order to define temperature structures for OTEC areas of interest, Ocean Data Systems, Inc. (ODSI) has developed computer data files of all unclassified soundings available. The primary sources for the data were NOAA's National Oceanographic Data Center, and the U.S. Navy's Fleet Numerical weather Central. The files were updated in September 1978. Included in the data base were mechanical bathythermographs (MBT), expendable bathythermographs (XBT), salinity temperature depth systems (STD), and Nansen casts.

Under U.S. Department of Energy, Division of Solar Energy Contract No. EY-78-C-2989, ODSI has analyzed the monthly temperature structure for ten different geographical areas. The ten sites selected for study by the Department of Energy are: 


\section{Latitude Longitude}

Bottom Mounted

or

Shore Plants: Dampier Land $13-18^{\circ} \mathrm{S} \quad 118-121^{\circ} \mathrm{E}$

\begin{tabular}{|c|c|c|c|}
\hline & Manila & $14-16^{\circ} \mathrm{N}$ & $118-120^{\circ} \mathrm{E}$ \\
\hline & Guam & $12-15^{\circ} \mathrm{N}$ & $142-146^{\circ} \mathrm{E}$ \\
\hline & Ivory Coast & $3-6^{\circ} \mathrm{N}$ & $3-8^{\circ} \mathrm{W}$ \\
\hline & Jakarta & $6-9^{\circ} \mathrm{S}$ & $104-109^{\circ} \mathrm{E}$ \\
\hline & Mexico & $20-23^{\circ} \mathrm{N}$ & $105-110^{\circ} \mathrm{W}$ \\
\hline & Sri Lanka & $5-10^{\circ} \mathrm{N}$ & $78-83^{\circ} \mathrm{E}$ \\
\hline & Mombasa & $5^{\circ} \mathrm{S}-3^{\circ} \mathrm{N}$ & $40-45^{\circ} \mathrm{E}$ \\
\hline hip Plants: & No. 1 & $5-10^{\circ} \mathrm{N}$ & $90-95^{\circ} \mathrm{W}$ \\
\hline & No. 2 & $13-15^{\circ} \mathrm{N}$ & $75-80^{\circ} \mathrm{W}$ \\
\hline
\end{tabular}

The location of these sites is shown on the following map; a separate report was produced for each site.

For each area, the most probable temperature structures were determined. When the most probable temperature soundings were plotted, some month to month variability was present which was caused by the non-uniform data sample rather than by real changes in the ocean. These short-period time variations were removed by a filtering process described by Wolff, et al (1977), [44]. Availability of cold and warm water was examined at each site. In addition to warm and cold water availability, there are other requirements for the continuous operation of an OTEC plant. An adequate temperature differential $(\Delta T)$ is the primary need. A $\Delta T$ greater than $16.7^{\circ} \mathrm{C}\left(30^{\circ} \mathrm{F}\right)$ for the coldest month of the year would enable year round operation. The annual mean $\Delta \mathrm{T}$ for 


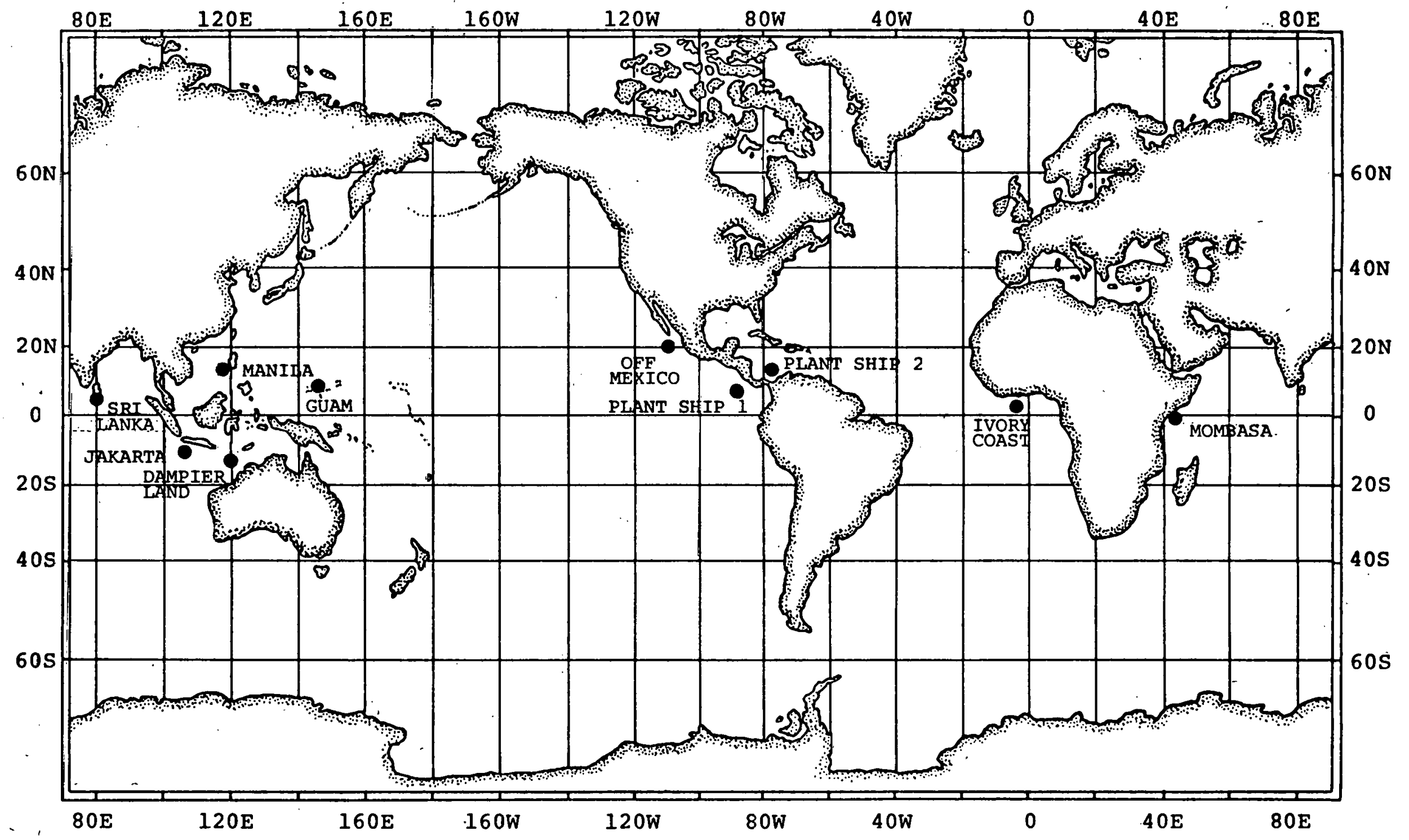

LOCATION OF THE TEN SELECTED SITES 
a site should equal or exceed $20^{\circ} \mathrm{C}\left(36^{\circ} \mathrm{F}\right)$. Besides the thermal resource, there are other operational requirements. . Bottom depth should be less than 1500 meters for mooring. Minimum distances offshore to 1000-meter depth is another important consideration for shore plants. Currents should be sufficient to guarantee good cold/warm water sources and to provide for dispersion of modified water. Desirable sites also have light winds, minimum sea and swell, and the lack of severe storms. These parameters have been examined for each site. The following tables summarize snme of the key site parameters for each location. 
SUMMARY OF SITE PARAMETERS

\begin{tabular}{|c|c|c|c|c|c|c|}
\hline Site & $\begin{array}{c}\text { Monthly Mean } \\
\text { Temperature }\left({ }^{\circ} \mathrm{C}\right) \\
\text { Surface Range } \\
\end{array}$ & \begin{tabular}{|c|} 
Monthly Mean \\
Temperature $\left({ }^{\circ} \mathrm{C}\right)$ \\
$1000 \mathrm{M}$ \\
\end{tabular} & $\begin{array}{c}\text { Annual } \\
\text { Mean } \\
\Delta \mathrm{T}\left({ }^{\circ} \mathrm{C}\right) 500 \mathrm{M} \\
\end{array}$ & $\begin{array}{c}\text { Annual } \\
\text { Mean } \\
\Delta \mathrm{T}\left({ }^{\circ} \mathrm{C}\right) 1000 \mathrm{M} \\
\end{array}$ & $\begin{array}{c}\text { Coldest Monthly } \\
\text { Mean } \Delta \mathrm{T}\left({ }^{\circ} \mathrm{C}\right) \\
500 \mathrm{M} \\
\end{array}$ & $\begin{array}{c}\text { Coldest Monthly } \\
\text { Mean } \Delta \mathrm{T}\left({ }^{\circ} \mathrm{C}\right) \\
1000 \mathrm{M} \\
\end{array}$ \\
\hline ' & & & & & & \\
\hline Mombasa $\mathrm{N}$ & $25.4-28.7$ & $7.2-7.5$ & 17.0 & 19.5 & 15.6 & $18.2^{-}$ \\
\hline Mombasa $\mathrm{S}$ & $25.5-28.4$ & $6.3-7.0$ & 18.0 & 20.2 & 15.9 & 18.5 \\
\hline Jakarta & $27.2-29.0$ & $5.1-5.6$ & 19.1 & 22.8 & 17.9 & 21.9 \\
\hline Manila & $27.1-29.5$ & $4.4-4.6$ & 20.0 & 24.0 & 18.6 & 22.6 \\
\hline Guam & $27.7-29.2$ & $4.3-4.4$ & 21.1 & 24.1 & 20.4 . & 23.4 \\
\hline Off Mexico & $22.5-28.0$ & $4.4-4.5$ & 17.6 & 20.9 & 14.9 & 18.0 \\
\hline $\begin{array}{c}\text { Plant Ship } \\
\text { Pacific }\end{array}$ & $27.1-28.5$ & $4.6-4.8$ & 19.4 & 22.8 & 18.1 & 21.7 \\
\hline Ivory Coast & $24.3-28.1$ & 4.5 & 19.2 & 22.1 & 16.8 & 19.7 \\
\hline
\end{tabular}


SUMMARY OF SITE PARAMETERS

\begin{tabular}{|c|c|c|c|c|c|}
\hline SITE & $\begin{array}{l}\text { RANGE DESTANCE } \\
\text { (IN KILOMETERS) } \\
\text { TO SHORE FROM } \\
\text { IOOC ME LERS }\end{array}$ & $\begin{array}{l}\text { MONTHLY MEAN } \\
\text { MIYED LAYER } \\
\text { DEFTH METERS }\end{array}$ & $\begin{array}{l}\text { MONTHLY MEAN } \\
\text { SURFACE CURRENTS } \\
\text { (CM/SEC) }\end{array}$ & $\begin{array}{l}\text { SEA STATE MAX } \\
\& \text { OF TIME }>3 \\
\text { METERS }\end{array}$ & $\begin{array}{l}\text { NUMBER TROPICAL } \\
\text { CYCLONES PER } \\
\text { YEAR }\end{array}$ \\
\hline SRI LANKA & $22-55$ & $30-80$ & $25-62$ & 3 & $0.2-1.2$ \\
\hline MOMBASA & $\equiv 3-130$ & $30-90$ & $30-62$ & 2 & $0.0-0.1$ \\
\hline JAKAR_A & $18-6 C$ & $55-80$ & $25-52$ & 2 & $0.0-0.1$ \\
\hline DAMPIER LAND & $265-417$ & $3 c-80$ & $25-47$ & 4 & $0.4-1.2$ \\
\hline MANILA & . $\quad 6-8 \bar{z}$ & $20-80$ & $30-52$ & 5 & $4.0-6.0$ \\
\hline GUAM & $7-1 \varepsilon$ & $60-120$ & $30-47$ & 5 & $2.0-3.0$ \\
\hline OFF MEXICO & $5-104$ & $10-30$ & $25-31$ & 2 & $0.6-4.0$ \\
\hline $\begin{array}{l}\text { PLANT SHIP } \\
\text { PACIFIC }\end{array}$ & - & $0-30$ & $30-52$ & 2 & $0.0-2.0$ \\
\hline IVORY COAST & $33-52$ & $0-30$ & $25-31$ & 2 & $0.0-0.1$ \\
\hline $\begin{array}{l}\text { PLANT SHIP } \\
\text { CARIBEEAN }\end{array}$ & - & $40-110$ & ${ }^{1} 30-62$ & 3 & $0.6-1.2$ \\
\hline
\end{tabular}




\section{EXECUTIVE SUMMARY}

The thermal resource off the Ivory Coast is quite good for OTEC purposes. There are consistently large $\Delta T$ (surface temperature - temperature at depth) values throughout the year. The mean $\Delta \mathrm{T}$ at 1000 meters is approximately $22^{\circ} \mathrm{C}$. A mean annual $\Delta \mathrm{T}$ of $20^{\circ} \mathrm{C}$ can be reached at a depth of only 600 meters. The thermal resource for the coldest month of the year is also adequate at 600 meters.

Water 1000 meters deep is available within $35 \mathrm{kilo-}$ meters from the shore. The waters south of the Ivory Coast do not have a particularly good mixed layer depth. Strong winds and tropical storms are a rare occurrence. Similarly, the sea and swell conditions do not present a probiem for OTEC development in this area. Currents are generally moderate, although occasional periods of weak currents less than $1 / 4$ knot can be expected. The consistently large thermal resource without major environmental problems recommends this location as a potential OTEC site. 


\section{BATHYMETRY}

Figure II-l shows the location of the potential site off the Ivory coast. The study area extended from $3-6^{\circ}$ North latitude and $3-8^{\circ}$ West longitude. The rough bathymetry for the area is shown in Figure II-2 (from the Defense Mapping Agency, 1974, [13]). The latter figure shows that distances to water of acceptable depth do not vary greatly.

Table II-1 summarizes distances offshore to selected depths.

TABLE II-I: DISTANCES TO SELECTED DEPTHS OFF THE IVORY COAST (Defense Mapping Àgency, 1974, [13] ?

\begin{tabular}{|ccc|}
\hline $\begin{array}{c}\text { DEPTH } \\
\text { (Meters) }\end{array}$ & $\begin{array}{c}\text { CLOSEST DISTANCE } \\
\text { (Kilometers) }\end{array}$ & $\begin{array}{c}\text { FARTHEST DISTANCE } \\
\text { (Kilometers) }\end{array}$ \\
\hline 100 & 7.5 & 26.0 \\
500 & 24.0 & 41.0 \\
1000 & 33.3 & 52.0 \\
1500 & 35.2 & 56.0 \\
\hline
\end{tabular}




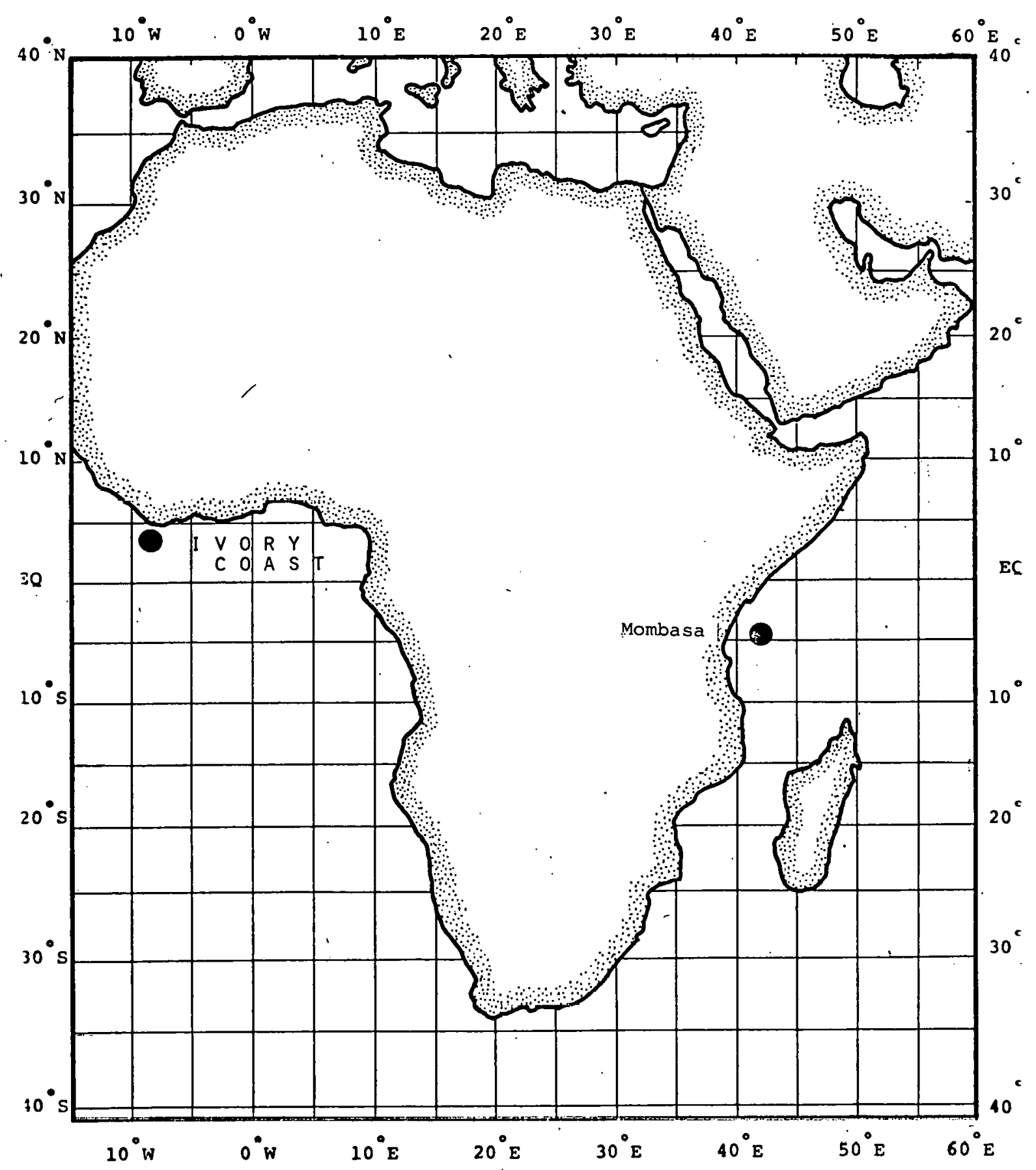

FIGURE II-1: LOCATOR CHART SHOWING THE LOCATION OF THE. - tVRRY conast nTES GTTE. 


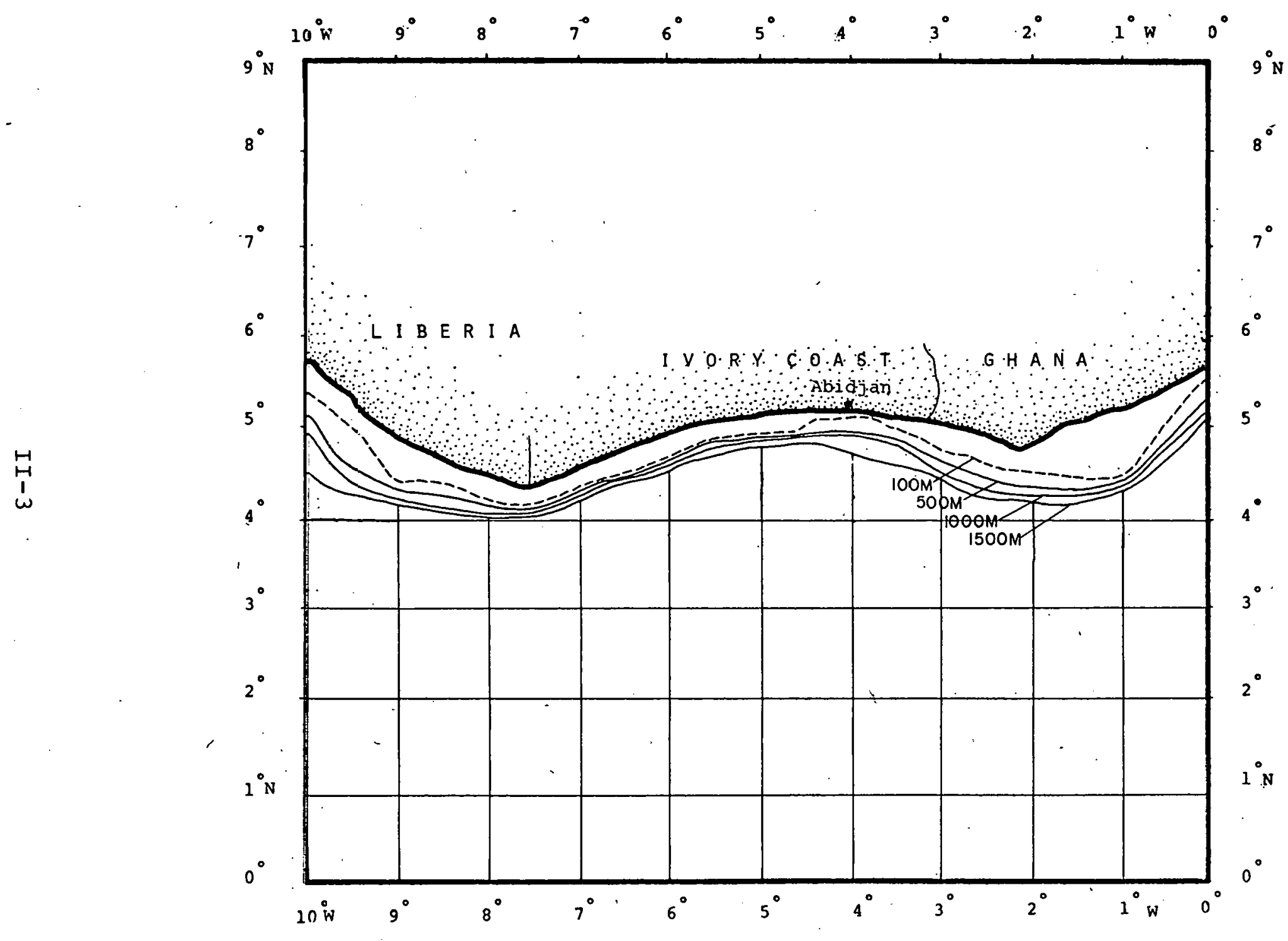

FIGURE II-2:. CHART SHOWING ROUGH BATHYMETRY OFF THE IVORY COAST (From Defense Mapping Agency, 1974, [113]). 
III. THERMAL RESOURCE

Table III-I represents the most probable temperature profile $\left({ }^{\circ} \mathrm{C}\right)$ for the Ivory Coast site. There were observations in Ocean Data Systems' master data file for all months of the year at every depth. Some short-period variations in the temperature with time were removed using a filtering process described by Wolff, et al (1977), [ㄴ4].

There was considerable variation in monthly mean sea surface temperatures at this site $\left(24.2^{\circ} \mathrm{C}\right.$ to $\left.28.0^{\circ} \mathrm{C}\right)$. Since temperatures at depths below 1000 meters were nearly constant, this means that values of $\Delta \mathrm{T}$ (surface temperature minus temperature at depth) also showed considerable variation. This can be seen in Table III-2 which shows the $\Delta T$ structure as a function of depth in meters. Figure III-I shows the same information plotted as contours of $\Delta T$ versus depth and month.'

Since this site has good access to deep cold waters from the Antarctic, the supply of cold water is excellent. An annual mean $\Delta \mathrm{T}$ of greater than $20^{\circ} \mathrm{C}$ is available at a depth of about 600 meters; however, due the seasonal variability, a depth of 1350 meters would have to be used Lu have a $20^{\circ} \mathrm{C} \Delta \mathrm{T}$ every month of the year. 
TABLE III-1: MONTHLY MOST PROBABLE TEMPERATURE $\left({ }^{\circ} \mathrm{C}\right)$

PROFILE IVORY COAST $3-6^{\circ} \mathrm{N} / 3-8^{\circ} \mathrm{W}$

\begin{tabular}{|c|c|c|c|c|c|c|c|c|c|c|c|c|c|}
\hline Depth & 1 & 2 & 3 & 4 & 5 & $\begin{array}{c}\text { Month } \\
6 \\
\end{array}$ & 7 & 8 & 9 & 10 & 11 & 12 & $\begin{array}{l}\text { Annual } \\
\text { Mean : }\end{array}$ \\
\hline 0 & 27.6 & 27.6 & 27.8 & 28.1 & 28.0 & 26.9 & 25.4 & 24.3 & 24.2 & 24.5 & 26.7 & 27.7 & 26.6 \\
\hline 50 & 19.2 & 19.2 & 20.1 & 21.1 & 22.1 & 20.7 & 19.3 & 17.5 & 17.8 & 19.4 & 20.3 & 19.2 & 19.7 \\
\hline 100 & 15.9 & 16.0 & 16.3 & 16.7 & 16.8 & 16.6 & 16.0 & 15.7 & 15.9 & 16.2 & 16.3 & 16.1 & 16.2 \\
\hline 150 & 14.8 & 14.9 & 15.0 & 15.3 & $15 . ?$ & 15.1 & 14.8 & 14.6 & 14.8 & 15.0 & 15.1 & 14.9 & 15.0 \\
\hline 200 & 13.9 & 14.0 & 14.0 & 14.2 & 14.1 & 13.9 & 13.6 & 13.5 & 13.8 & 14.0 & 14.1 & 14.1 & 13.9 \\
\hline 250 & 12.6 & 12.5 & 12.5 & 12.5 & 12.5 & 12.2 & 12.0 & 11.9 & 12.3 & 12.6 & 12.8 & 12.7 & 12.4 \\
\hline 300 & 11.1 & 11.0 & 11.0 & 10.9 & 10.9 & 10.6 & 10.5 & 10.5 & 10.8 & 11.2 & 11.4 & 11.3 & 10.9 \\
\hline 350 & 9.9 & 9.7 & 9.6 & 9.6 & 9.6 & 9.4 & 9.4 & 9.4 & 9.7 & 10.0 & 10.1 & 10.1 & 9.7 \\
\hline 400 & 8.9 & 8.8 & 8.7 & 8.6 & 8.6 & 8.5 & 8.5 & 8.5 & 8.6 & 9.0 & 9.1 & 9.0 & 8.7 \\
\hline 450 & 8.1 & 8.0 & 7.9 & 7.9 & 7.8 & 7.8 & 1.8 & 7.9 & 8.0 & 8.2 & 8.3 & 8.2 & 8.0 \\
\hline 500 & 7.5 & 7.4 & 7.3 & 7.2 & 7.2 & 7.2 & 7.2 & 7.3 & 7.4 & 7.5 & 7.5 & 7.5 & 7.4 \\
\hline 550 & 6.9 & 6.9 & 6.8 & 6.7 & 6.7 & 6.7 & 6.7 & 6.8 & 6.9 & 6.9 & 6.9 & 6.9 . & 6.8 \\
\hline 600 & 6.4 & 6.4 & 6.3 & 6.2 & 6.2 & 6.3 & 6.3 & 6.3 & 6.4 & 6.4 & 6.4 & 6.4 & 6.3 \\
\hline 650 & 6.0 & 6.0 & 5.9 & 5.8 & 5.8 & 5.8 & 5.8 & 5.9 & 5.9 & 6.0 & 5.9 & 6.0 & 5.9 \\
\hline 700 & 5.7 & 5.7 & 5.5 & 5.5 & 5.5 & 5.5 & 5.5 & 5.6 & 5.6 & 5.6 & 5.6 & 5.6 & 5.6 \\
\hline 750 & 5.4 & 5.4 & 2 & 5.2 & 5.2 & 2 & 5.2 & 5.3 & 5.3 & 5.3 & 5.3 & 5.3 & 5.3 \\
\hline 800 & 5.1 & 5.0 & .0 & 5.0 & 5.0 & 5.0 & 5.0 & 5.0 & 5.1 & 5.1 & 5.1 & 5.1 & 5.1 \\
\hline 850 & 4.9 & 4.9 & 4.8 & 4.8 & .4 .8 & 4.8 & 4.8 & 4.9 & 4.9 & 4.9 & 4.9 & 4.9 & 4.9 \\
\hline 900 & 4.8 & 4.8 & 4.7 & 4.7 & 4.6 & $4: 6$ & 4.7 & 4.7 & 4.8 & 4.8 & 4.7 & 4.8 & 4.7 \\
\hline 950 & 4.6 & 4.6 & 4.6 & 4.6 & 4.5 & 4,5 & 4.6 & 4.6 & 4.0 & 4.6 & 4.6 & 4.6 & 4.6 \\
\hline 1000 & 4.5 & 4.5 & 4.5 & 4.5 & 4.5 & 4.5 & 4.5 & 4.5 & 4.5 & 4.5 & 4.5 & 4.5 & 4.5 \\
\hline 1050 & 4.4 & 4.5 & 4.4 & 4.4 & 4.4 & 4.4 & 4.5 & 4.5 & 4.5 & 4,5 & 4.1 & 4.5 & 4.5 \\
\hline 1100 & 4.4 & 4.4 & 4.4 & 4.4 & 4.4 & 4.4 & 4.4 & 4.4. & 4.4 & 4.4 & 4.4 & 4.4 & 4.4 \\
\hline 1150 & 4.4 & 4.4 & 4.4 & 4.4 & 4.4 & 4.4 & 4.4 & 4.4 & 4.4 & 4.4 & $4 . \dot{4}$ & 4.4 & 4.4 \\
\hline 1200 & 4.3 & 4.3 & 4.3 & 4.3 & 4.3 & 4.3 & 4.3 & 4.3 & 4.3 & 4.3 & 4.3 & 4.3 & 4.3 \\
\hline 1250 & 4.3 & 4.3 & 4.3 & 4.3 & 4.3 & 4.3 & 4.3 & 4.3 & 4.3 & 4.3 & 4.3 & 4.3 & 4.3 \\
\hline 1300 & 4.2 & 4.3 & 4.3 & 4.3 & 4.2 & 4.3 & 4.3 & 4.3 & 4.3 & 4.3 & 4.2 & 4.2 & 4.3 \\
\hline 1350 & 4.2 & 4.2 & 4.2 & 4.2 & 4.2 & 4.2 & 4.2 & 4.2 & 4.2 & 4.1 & 4.1 & 4.2 & $4: 2$ \\
\hline 1400 & 4.1 & 4.1 & 4.1 & 4.1 & 4.1 & 4.1 & 4.1 & 4.1 & 4.1 & 4.1 & 4.1 & 4.1 & 4.1 \\
\hline 1450 & 4.1 & 4.1 & 4.1 & 4.1 & 4.1 & 4.1 & 4.1 & 4.1 & 4.1 & 4.1 & 4.1 & 4.1 & 4.1 \\
\hline 1500 & 4.1 & 4.1 & 4.1 & 4.0 & 4.1 & 4.1 & 4.1 & 4.0 & 4.1 & 4.0 & 4.0 & $4: 0$ & 4.1 \\
\hline
\end{tabular}




\begin{tabular}{|c|c|c|c|c|c|c|c|c|c|c|c|c|c|}
\hline \multirow{2}{*}{ Depth } & \multicolumn{12}{|c|}{ Month } & \multirow{2}{*}{$\begin{array}{l}\text { Annual } \\
\text { Mean }\end{array}$} \\
\hline & 1 & 2 & 3 & 4 & 5 & 6 & 7 & 8 & 9 & 10 & 11 & 12 & \\
\hline 50 & 8.4 & 8.4 & 7.7 & 7.0 & 5.9 & 6.2 & 6.1 & 6.8 & 6.4 & 5.1 & 6.4 & 8.5 & 6.9 \\
\hline 100 & 11.7 & 11.6 & 11.5 & 11.4 & 11.2 & 10.3 & 9.4 & 8.6 & 8.3 & $8: 3$ & 10.4 & 11.6 & 10.4 \\
\hline 150 & 12.8 & 12.7 & 12.8 & 12.8 & 12.7 & 11.8 & 10.6 & 9.7 & 9.4 & 9.5 & 11.6 . & 12.8 & 11.6 \\
\hline 200 & 13.7 & 13.6 & 13.8 & 13.9 & 13.9 & 13.0 & 11.8 & 10.8 & 10.4 & 0.5 & 12.6 & 13.6 & 12.6 \\
\hline 250 & 15.0 & 15.1 & 15.3 & 15.6 & 15.5 & 14.7 & 13.4 & 12.4 & 11.9 & 11.9 & 13.9 & 15.0 & 14.1 \\
\hline 300 & 16.5 & 16.6 & 16.8 & 17.2 & 17.1 & 16.3 & 14.9 & 13.8 & 13.4 & 13.3 & 15.3 & 16.4 & 15.6 \\
\hline 350 & 17.7 & 17.9 & 18.2 & 18.5 & 18.4 & 17.5 & 16.0 & 14.9 & 14.5 & 14.5 & 16.6 & 17.6 & 16.9 \\
\hline 400 & 18.7 & 18.8 & 19.1 & 19.5 & 19.4 & 18.4 & 16.9 & 15.8 & 15.6 & 15.5 & 17.6 & 18.7 & 17.8 \\
\hline 450 & 19.5 & 19.6 & 19.9 & 20.2 & 20.2 & 19.1 & 17.6 & 16.4 & 16.2 & 16.3 & 18.4 & 19.5 & 18.6 \\
\hline 500 & 20.1 & 20.2 & 20.5 & 20.9 & 20.8 & 19.7 & 18.2 & 17.0 & 16.8 & 17.0 & 19.2 & 20.2 & 19.2 \\
\hline 550 & 20.7 & 20.7 & 21.0 & 21.4 & 21.3 & 20.2 & 18.7 & 17.5 & 17.3 & 17.6 & 19.8 & 20.8 & 19.8 \\
\hline 600 & 21.2 & 21.2 & 21.5 & 21.9 & 21.8 & 20.6 & 1 & 18.0 & 17.8 & 8.1 & 20.3 & 21.3 & 20.2 \\
\hline 650 & 21.6 & 21.6 & 21. & 22.3 & 22.2 & 21.1 & 19.6 & 18.4 & 18.3 & 8.5 & 20.8 & 21.7 & 20.7 \\
\hline 700 & 21.9 & 21.9 & 22.3 & 22.6 & 22.5 & 21.4 & 19.9 & 18.7 & 18.6 & 18.9 & 21.1 & 22.1 & 21.0 \\
\hline 750 & 22.2 & 22.2 & 22.6 & 22.9 & 22.8 & 21.7 & 20.2 & 19.0 & 18.9 & 19.2 & 21.4 & 22.4 & 21.3 \\
\hline 800 & 22.5 & 22.6 & 22.8 & 23.1 & 23.0 & 21.9 & 20.4 & 19.3 & 19.1 & 19.4 & 21.6 & 22.6 & 21.5 \\
\hline 850 & 22.7 & 22.7 & 23.0 & 23.3 & 23.2 & 22.1 & 20.6 & 19.4 & 19.3 & 19.6 & 21.8 & 22.8 & 21.7 \\
\hline 900 & 22.8 & 22.8 & 23.1 & 23.4 & 23.4 & 22.3 & 20.7 & 19.1 & 19.4 & 19.7 & 22.0 & 22.9 & 21.8 \\
\hline 950 & 23.0 & 23.0 & 23.2 & 23.5 & 23.5 & 22.4 & 20.8 & 19.7 & 19.6 & 19.9 & 22.1 & 23.1 & 22.0 \\
\hline 1000 & 23.1 & $23 . i$ & 23.3 & 23.6 & 23.5 & 22.4 & 20.9 & 19.8 & 15 & 20.0 & 22.2 & 2 & 22.1 \\
\hline 1050 & 23.2 & 23.1 & 23.4 & 23.7 & 23.6 & 22.5 & 20.9 & 19.8 & 19.7 & 0.0 & 22.3 & 23.2 & $22: 1$ \\
\hline 1100 & 23.2 & 23.2 & 23.4 & 23.7 & 23.6 & 22.5 & 21.0 & 19.9 & 19.8 & 20.1 & 22.3 & 23.3 & 22.2 \\
\hline 1150 & 23.2 & 23.2 & 23.4 & 23.7 & 23.6 & 22.5 & 21.0 & 19.9 & 19.8 & 20.1 & 22.3 & 23.3 & 22.2 \\
\hline 1200 & 23.3 & 23.3 & 23.5 & 23.8 & 23.7 & 22.6 & 21.1 & 20.0 & 19.9 & 20.2 & 22.4 & 23.4 & 22.3 \\
\hline 1250 & 23.3 & 23.3 & 23.5 & 23.8 & 23.7 & 22.6 & 21.1 & 20.0 & 19.9 & 20.2 & 22.4 & 23.4 & .3 \\
\hline 1300 & 23.4 & 23.3 & 23.5 & 23.8 & 23.8 & 22.6 & 21.1 & 20.0 & 19.9 & 20.2 & 22.5 & 23.5 & 22.3 \\
\hline 1350 & 23.4 & 23.4 & 23.6 & 23.9 & 23.8 & 22.7 & 21.2 & 20.1 & 20.0 & 20.4 & 22.6 & 23.5 & 22.4 \\
\hline 1400 & 23.5 & 23.5 & 23.7 & 24.0 & 23.9 & 22.8 & 21.3 & 20.2 & 20.1 & 20.4 & 22.6 & 23.6 & 22.5 \\
\hline 1450 & 23.5 & 23.5 & 23.7 & 24.0 & 23.9 & 22.8 & 21.3 & 20.2 & 20.1 & 20.4 & 22.6 & 23.6 & 22.5 \\
\hline 1500 & 23.5 & 23.5 & 23.7 & 24.1 & 23.9 & 22.8 & 21.3 & 20.3 & 20.1 & 20.5 & 22.7 & 23.7 & 22.5 \\
\hline
\end{tabular}




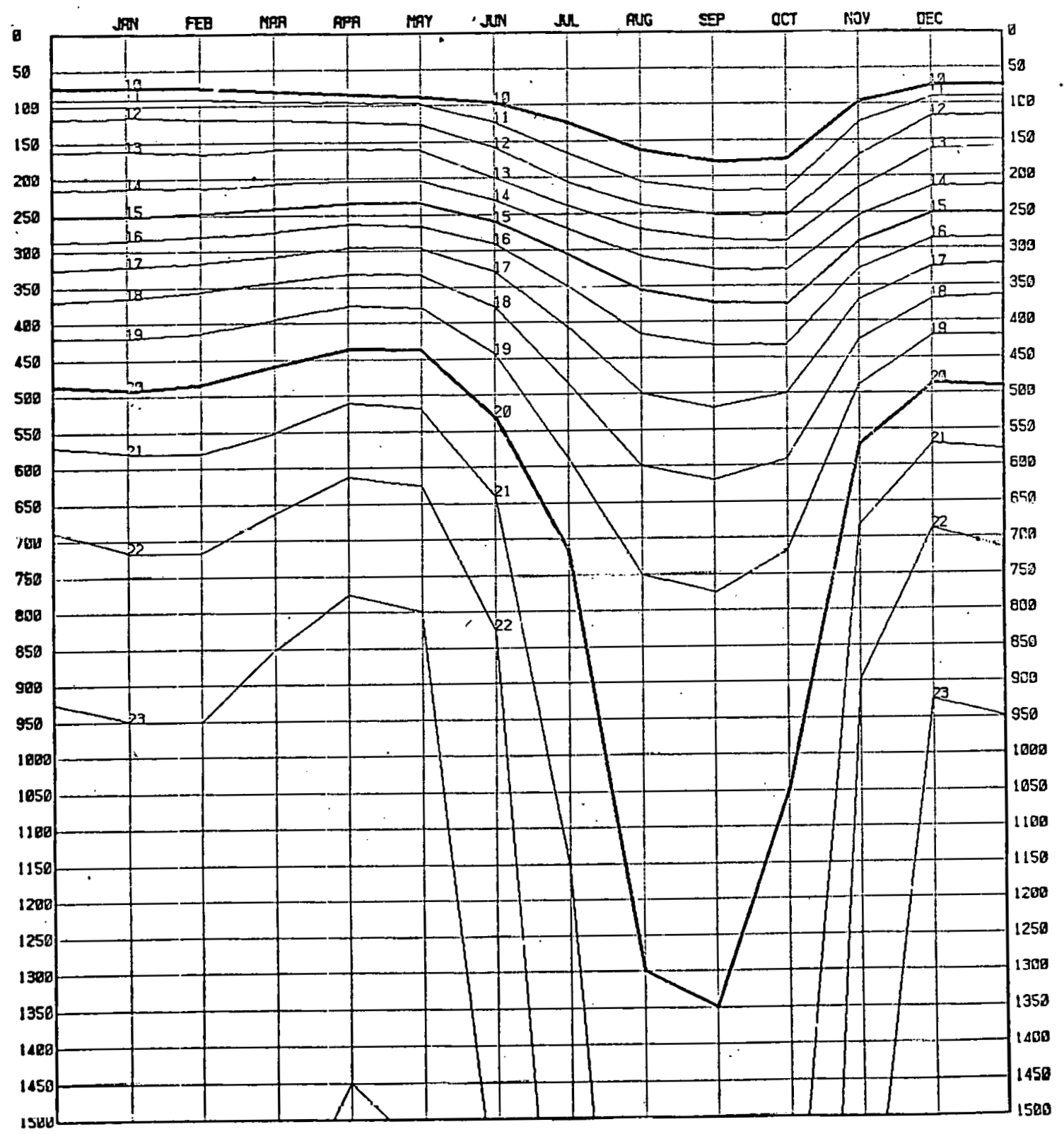

FIGURE III-1: CHART SHOWING MONTHLY $\triangle T$ CONTOURS AT A DEPTH RESULU'TION OF 50 METERS OFF THE IVORY COAST $\left(3-6^{\circ} \mathrm{N}, 3-8^{\circ} \mathrm{W}\right)$. 
IV. MIXED LAYER DEPTH

For OTEC purposes, the Mixed Layer Depth (MLD) is defined as the depth at which the temperature first becomes colder than the surface temperature by $1^{\circ} \mathrm{C}$. A well-defined upper mixed layer is favorable for OTEC operations. Ideally, the layer should be deep enough to insure a good supply of warm water but not so deep as to lead to the possibility of recirculation of discharged, modified water.

The vertical temperature gradient is very strong off the Ivory Coast in the first 50 meters below the surface. Because of the light winds and lack of wave mixing from January through March, there is often no MLD during this period. When there is an upper mixed layer, it is generally very shallow. 


\section{WINDS AND STORMS}

The site off the Ivory Coast is located between $3-6^{\circ}$ North latitude. This area is part of the doldrums, characterized by calms or very light winds. Tropical storms are rare in the area. In January, $24 \%$ of the time the winds are calm. Similarly, the winds for spring, summer, and fall periods are generally light. The following figures demonstrate the weak winds in the area:

June

$5 \%$ calm

55 4-10 knots

$25 \%$ 11-16 knots

10-15영 17-27 knots

$1 \% \geq 28$ knots

\section{September}

$8 \% \mathrm{calm}$

$62 \%$ 4-10 knots

20\% 10-16 knots

8응 17-27 knots

The prevailing wind direction throughout the year is from the south. This data is from the Oceanographic Atlas of the North Atlantic Ocean (1963), [37].

Tables $V-1$ through $V-4$ with data from the Weather Bureau's Atlas of Climatic Charts of the Oceans provides information on the winds for each of the ten sites under this contract. These tables show that winds of near gale force or greater are unusual for the Ivory Coast site.

Figure V-1 adapted from H.L. Crutcher and R.G. Quayle (1974), [ 7], shows the preferred annual storm tracks this area. 
TABLE $V-I$

RESULTANT NINDS

Average Wind Velocity in vieters per Second

\begin{tabular}{|l|c|c|c|c|}
\hline Location & Dec-Jan-Feb & Mar-Apr-May & Jun-Jul-Aug & Sep-Oct-Nov \\
\hline Ivory Coast & $3.1-4.1$ & $3.1-4.1$ & $4.1-5.1$ & $.4 .1-5.1$ \\
Mombasa & $5.1-6.2$ & $4.1-5.1$ & $6.2-7.2$ & $4.1-5.1$ \\
Sri Lanka & $4.1-5.1 W$ & $4.1-5.1$ & $6.2-7.2 \mathrm{~W}$ & $5.1-6.2$ \\
& $5.1-6.2 \mathrm{E}$ &. & $7.2-8.2 \mathrm{E}$ & \\
Jakartá & 4.1 & $3.1-4.1$ & $5.1-6.2$ & $5.1-6.2$ \\
Dampier Land & $4.1-5.1$ & $4.1-6.2$ & $5.1-6.2$ & $1.1-5.1$ \\
Philippines & $5.1-6.2$ & $3.1-4.1$ & 4.1 & $6.2-7.2$ \\
Guam & $5.1-6.2$ & $4.1-5.1$ & $4.1-5.1$ & $4.1-5.1$ \\
Off Mexico & $3.1-4.1$ & $2.1-4.1$ & $2.1-4.1$ & $4.1-5.1$ \\
Plant Ship & $3.1-4.1$ & $3.1-4.1$ & $4.1-5.1$ & $4.1-6.2$ \\
Pacific & & $5.1-6.2$ & $6.2-8.2$ & $4.1-5.1$ \\
Plant Ship & $6.2-7.2$ & & & \\
Caribbean & & & & \\
\hline
\end{tabular}


TABLE V- 2

FREQUENCY AND PEFCENTAGE OF MODERATE GALES AND STRONGER WINDS,

BEAUFORT FORCE 7 AND HIGIER

( $\geq 14.4$ neters per second)

\begin{tabular}{|c|c|c|c|c|c|c|c|c|c|c|}
\hline MONTH & $\begin{array}{l}\text { IVORY } \\
\text { COAST } \\
\end{array}$ & MOIMAASA & $\begin{array}{c}\text { SRI } \\
\text { LANKA }\end{array}$ & JAKAITA & $\begin{array}{c}\text { DAMP I ER } \\
\text { LAND } \\
\end{array}$ & PHILLIPINES & GUAM & $\begin{array}{c}\text { OFF } \\
\text { MEXICO }\end{array}$ & $\begin{array}{c}\text { PLAIN } \\
\text { SHIP } \\
\text { (PACIFIC) }\end{array}$ & $\begin{array}{c}\text { PLANT } \\
\text { SHIP } \\
\text { (CARIBN) } \\
\end{array}$ \\
\hline JAN & 0 * & $0-1$ & 0 & 0 & 0 & $1-5$ & $1-5$ & $1-5$ & 0 & 5 \\
\hline FEB & 0 & 0. & 1 & $1-5$ & $1-5$ & 5 & $1-2$ & $1-2$ & 0 & 5 \\
\hline MAR & $1-2$ & 0 & 0 & $1-2$ & $1-2$ & 1 & 0 & $1-2$ & 0 & $1-5$ \\
\hline$\grave{\lambda P R}$ & 0 & 0 & 0 & $1-2$ & 0 & 1 & $1-5$ & 1 & $0:$ & $1-5$ \\
\hline$\because A A Y$ & 0 & 1 & $1-5$ & 1 & 0 & $1-5$ & 0 & 0 & 0 & 1 \\
\hline JUN & 0 & $5-10$ & $5-10$ & $1-5$ & 0 & $1-5$ & 0 & 1 & 0 & $1-5$ \\
\hline JUL & 0 & $\begin{array}{r}5-10 \mathrm{~N} \\
10-20 \mathrm{~S}\end{array}$ & $1-5$ & $1-5$ & $1-5$ & 5 & 0 & 0 & 0 & $1-5$ \\
\hline AUG & 0 & $\begin{array}{l}1-5 S \\
5-15 N\end{array}$ & $1-5$ & 0 & 0 & $1-5$ & $1-5$ & $1-5$ & 0 & $1-5$ \\
\hline SEP & 0 & 0 & $1-5$ & $1-5$ & 0 & 5 & $1-5$ & 0 & 0 & 1 \\
\hline OCT & 0 & 0 & $\begin{array}{c}O W \\
1-5 E\end{array}$ & $1-5$ & 0 & $1-5$ & $1-2$ & -1 & 1 & $1-5$ \\
\hline NOV & 0 . & 0 & $1-5$ & $1-j$ & 0 & $\begin{array}{r}10-15 \mathrm{~N} \\
1-10 \mathrm{~S}\end{array}$ & $5-15$ & $I-5$ & $0-5$ & $1-5$ \\
\hline DEC & 0 & $1-2$ & $\begin{array}{c}\text { OW } \\
I-5 E\end{array}$ & $1-5$ & 0 & $\begin{array}{r}10-15 \mathrm{~N} \\
5-10 \mathrm{~S}\end{array}$ & $1-5$ & $1-5$ & 0 & $1-5$ \\
\hline
\end{tabular}

* $0=$ few or none. 
TABLE V- 3

PERCENTAGE OF WINDS WITH BEAUFORT FORCE \& AND HIGHER ( $\geq 17.5$ meters per second)

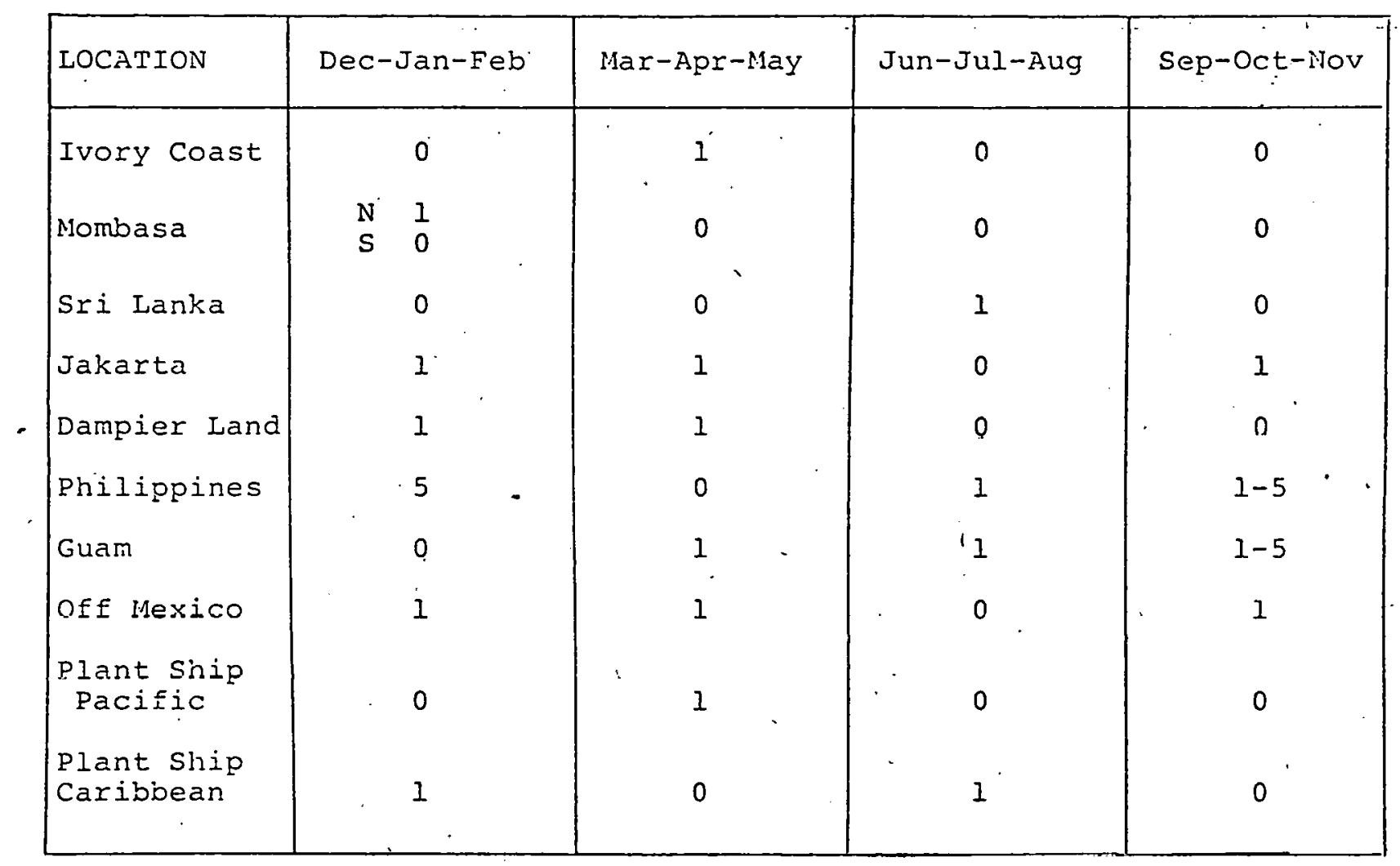


TABLE $V-4$

PREDOMINANT SURFACE WIND DIRECTION

\begin{tabular}{|c|c|c|c|c|c|c|c|c|c|c|}
\hline МоNтн & MOMBASR. & $\begin{array}{c}\text { SRI } \\
\text { LANKA }\end{array}$ & DAMPIER & JAKARTA & MANILA & GUAM & $\begin{array}{l}\text { MFF } \\
\text { MEXICO }\end{array}$ & $\begin{array}{c}\text { PLANA } \\
\text { SHIP } \\
\text { (PACIFIC) }\end{array}$ & $\begin{array}{l}\text { PLANT } \\
\text { SHAP } \\
\text { CCARID }\end{array}$ & \begin{tabular}{|l} 
IVORY \\
COAST
\end{tabular} \\
\hline JAN & $\downarrow$ & $\angle$ & ת & $\nu$ & $\downarrow$ & $\measuredangle$ & \pm & $\measuredangle$ & $\swarrow$ & $\uparrow \nearrow$ \\
\hline FEB & $\downarrow$ & $<$ & $>$ & $\rightarrow$ & $\swarrow$ & $\swarrow$ & $\downarrow$ & $\measuredangle$ & $\measuredangle$ & $\uparrow$ \\
\hline MAR & $<$ & ১০ & $\longrightarrow$ & $\longrightarrow$ & $\downarrow$ & $\downarrow$ & $\nu$ & $\measuredangle$ & $\leftarrow$ & $\uparrow$ \\
\hline${ }_{A P R}$ & $0^{\circ} \frac{\alpha}{\pi}$ & 果. & $\kappa$ & k & $\downarrow$ & $<$ & $\searrow$ & $r$ & $\leftarrow$ & $\uparrow$ \\
\hline MAY & $\uparrow \uparrow$ & $\nearrow$ & $\pi$ & $\kappa$ & 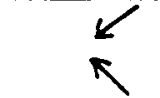 & 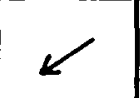 & $\searrow$ & $\pi$ & $\leftarrow$ & $\uparrow$ \\
\hline JCN & $\uparrow$ & 7 & $\pi$ & $\Omega$ & $\lambda$ & $\leftarrow$ & $\searrow$ & ${ }_{\uparrow}{ }_{\uparrow}$ & $\leftarrow$ & $\uparrow$ \\
\hline JUL & $\uparrow$ & $\lambda$ & $\pi$ & $\pi$ & $\nearrow$ & $\hat{i}$ & 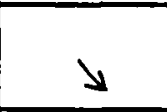 & $\uparrow$ & $\leftarrow$ & $\uparrow$ \\
\hline AUG & $\uparrow$ & $\lambda$ & $\pi$ & $\pi$ & 7 & $\leftarrow$ & $\downarrow$ & $\vec{\uparrow} \overrightarrow{ }$ & $\leftarrow$ & $\nearrow$ \\
\hline SEP & $\uparrow$ & $\rightarrow 0^{\lambda}$ & $\pi$ & $\pi$ & $v_{x}$ & $\leftarrow$ & $y$ & $\pi$ & $\leftarrow$ & $\uparrow$ \\
\hline OCT & $\uparrow$ & $\rightarrow$ & $\pi$ & $\pi$ & $<$ & $\measuredangle$ & $\downarrow$ & $\uparrow$ & $\leftarrow$ & $\uparrow$ \\
\hline Nov & $\frac{\alpha}{K}$ & $\rightarrow$ & $\vec{r}$ & ${ }^{\Delta} \pi$ & $<$ & $\downarrow$ & $\downarrow$ & $\begin{array}{ll}k \\
1\end{array}$ & $\leftarrow$. & $\uparrow$ \\
\hline $\mathrm{D} \Xi \mathrm{C}$ & $\ll$ & 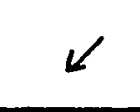 & $\nearrow$ & $\lambda$ & $\downarrow$ & $\swarrow$ & $\downarrow$ & $\leftarrow$ & $<$ & $\nearrow$ \\
\hline
\end{tabular}




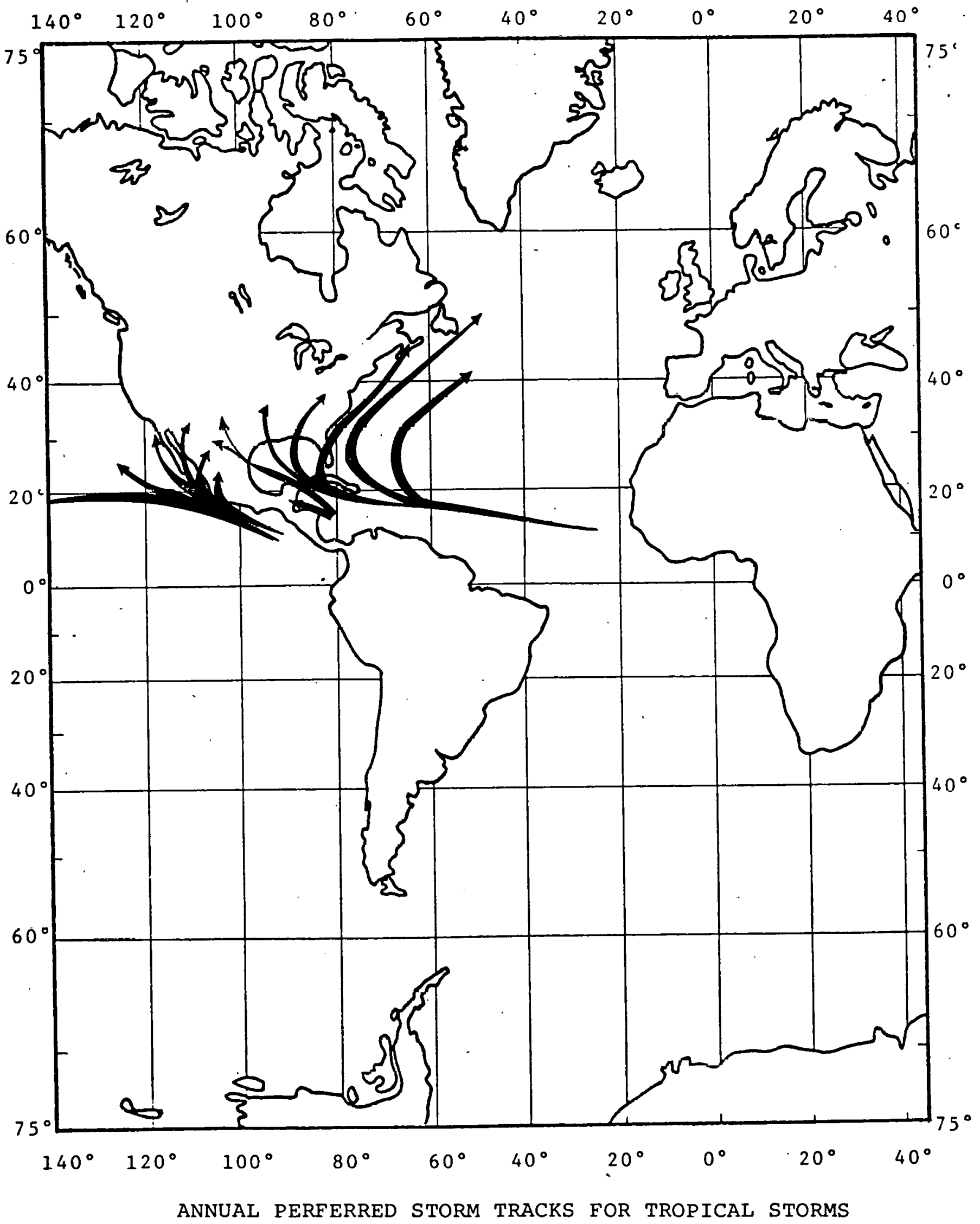

FIGURE $\mathrm{V}-1$ 
Figure V-2 from the same source provides the average number of tropical cyclones per $5^{\circ}$ square per year. As these figures show tropical storms and hurricanes are not a problem for the Ivory coast site. 


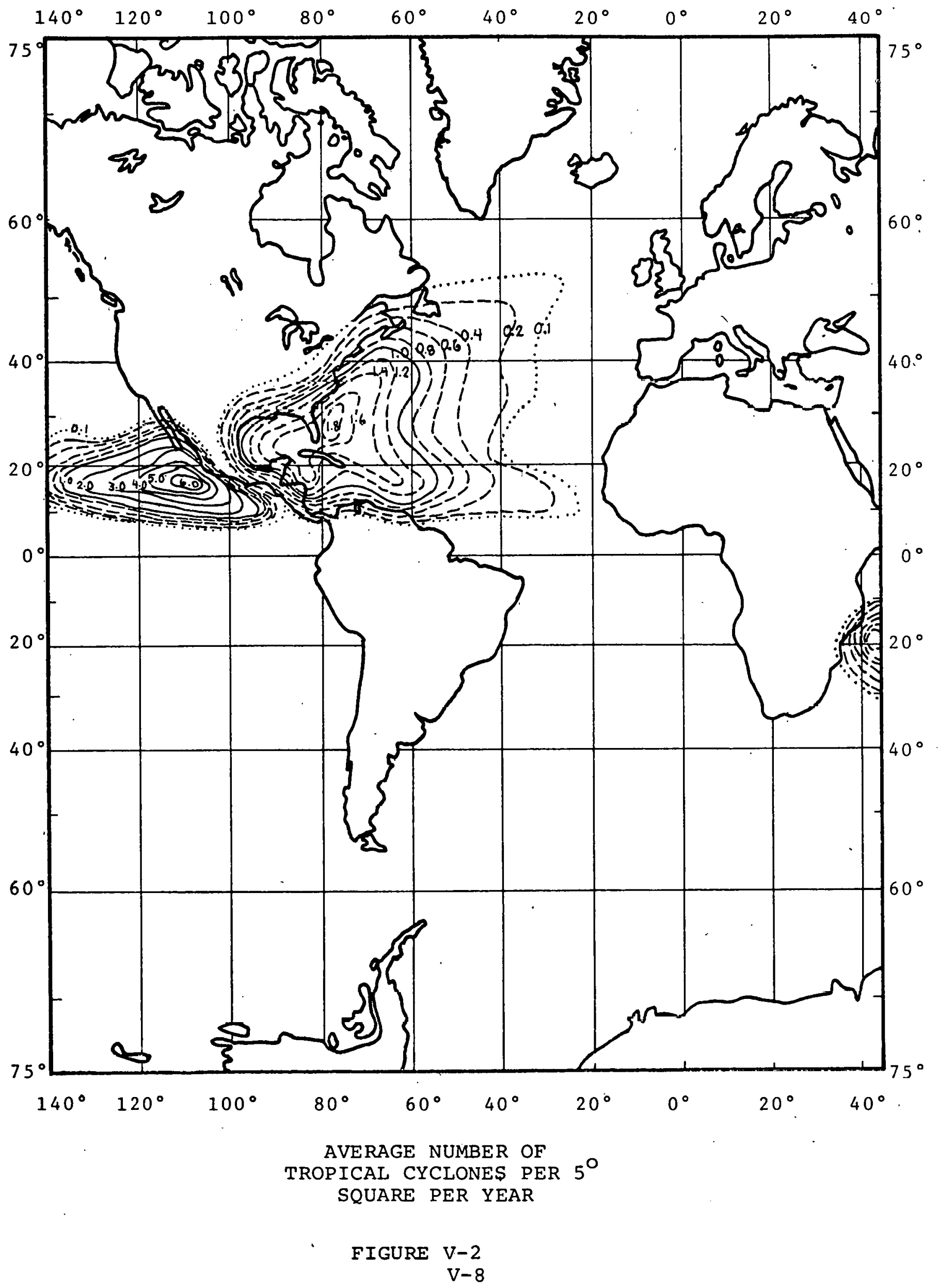


VI. SEA AIND SWELL CHARACTERISTICS

Table VI-1, taken from Ocean Wave Statistics; (1967), [19], presents a statistical breakdown showing the number of observations in various wave heights versus period categories. This summary is based upon actual ships' observations from a somewhat larger region south of the Ivory Coast, however, conditions over the area are represented as being homogeneous.

The predominant direction of the waves is from the south. This direction is constant sixty to eighty percent of the time. The roughest average sea conditions exist during June and July. Throughout the year, however, the state of the sea can be characterized as slight with waves less than one meter. Similarly, the low swell conditions predominate throughout the year with swells less than two meters. 
TABLE VI-1: STATISTICAL BREAKDOWN SHOWING NUMBER OF SHבPS OBSERVATIONS SOUTH OF THE IVORY COAST REPORTING VARIOUS HEIGH $/$ /PERIOD COMBINATIONS (ALI SEASONS: From Ocean. Wave Statistics $[1967)$, [19].

\begin{tabular}{|c|c|c|c|c|c|c|c|c|c|c|c|c|c|}
\hline $\begin{array}{l}\text { Wave Height } \\
\text { (meters) }\end{array}$ & $\mathrm{Ca} \mathrm{m}$ & $<5$ & $6-7$ & $\begin{array}{l}\text { Wave } \\
8-9\end{array}$ & $\begin{array}{l}\text { Period } \\
10-11\end{array}$ & $\begin{array}{c}\text { (Seconds) } \\
12-13\end{array}$ & $14-15$ & $16-17$ & $18-19$ & $20-21$ & $\begin{array}{r}\text { over } \\
22 \\
\end{array}$ & Total & $\begin{array}{l}\text { Percent of } \\
\text { Grand Total }\end{array}$ \\
\hline 0.25 & 1589 & 1484 & 41 & 15 & 5 & 4 & 7 & 1 & & 31 & 28 & 3208 & 16 \\
\hline 0.5 & 101 & 3338 & 319 & 99 & 49 & 18 & 8 & 4 & & 7 & 237 & 4180 & 20 \\
\hline 1.0 & 90 & 4665 & 1899 & 379 & 100 & 63 & 20 & 9 & 1 & 7 & 67 & 7300 & 35 \\
\hline 1.5 & 35 & 1330 & 1963 & 625 & 150 & 71 & 29 & 9 & 1 & & 6 & 4219 & 20 \\
\hline 2.0 & 9 & 171 & 535 & 381 & 130 & 28 & 17 & 4 & 3 & & 1 & 1279 & 6 \\
\hline 2.5 & 3 & 21 & 100 & 142 & 49 & 24 & 4 & & 1 & & & 344 & 2 \\
\hline 3.0 & & 5 & 17 & 25 & 19 & 11 & 3 & 1 & & & 1 & 82 & $<1$ \\
\hline 3.5 & & 4 & 2 & 10 & 4 & 1 & & & & & & 21 & $<1$ \\
\hline 4.0 & & & 2 & 2 & 1 & 2 & & & - & 2 & & 9 & $<1$ \\
\hline 4.5 & & 1 & & 6 & 1 & 1 & 2 & 2 & & 1 & & 14 & $<1$ \\
\hline 5.0 & 5 & $I$ & & & & & & & & & & 6 & $<1$ \\
\hline 5.5 & 1 & 1 & & & & & & & & & & 2 & $<I$ \\
\hline 6.0 & & 2 & & 1 & & & & 2 & & & & 5 & $<I$ \\
\hline 6.5 & & 1 & & 2 & & & 2 & & & & & 5 & $<1$ \\
\hline 7.0 & 1 & & & 1 & & & 3 & & & & & 2 & $<1$ \\
\hline TOTAL & 1834 & $1102 L$ & 2878 & 1688 & 508 & 223 & 92 & 32 & 6 & 48 & 340 & 20673 & \\
\hline $\begin{array}{l}\text { Percent } \\
\text { of Grand } \\
\text { Total }\end{array}$ & 9 & 53 & 24 & 8 & 2 & 1 & $<1$ & $<1$ & $e_{1}$ & $<1$ & 1 & & r. \\
\hline
\end{tabular}


VII. CURRENTS

The predominant current of the South Atlantic Ocean is the Benguela Current, which flows along the west coast of Africa. As the Benguela Current proceeds towards the equator, it leaves the coast and continues as the northern portion of the South Equatorial current which flows towards the west across the Atlantic, usually between $0^{\circ}$ and $20^{\circ}$ south. Three degrees north latitude is the northernmost boundary of the South Equatorial current. Thus, the region of this study is in the area of the Equatorial Countercurrent. The Guinea Current, which flows eastward off the coast of the Ivory Coast is a part of the undercurrent. The flow of the equatorial undercurrent can be quite strong. The Encyclopedia of Oceanography $(1966,[30])$ states that the flow can be up to $116 \mathrm{~cm} / \mathrm{sec}$ at the equator at the longitude of the Ivory Coast site. Between $3-6^{\circ} \mathrm{N}$ the current speeds are generally less thạn $50 \mathrm{~cm} / \mathrm{sec}$.

Hurricanes, while rare at this site, can cause changes in surface currents: Leipper's 1967 [21] study of Hurricane Hilda showed that a current had developed in the area transversed by the storm. Analysis of temperature - depth showed a current of approximately $50 \mathrm{~cm} / \mathrm{sec}$. A theoretical study by O'Brien and Reid (1967, [27]) states that hurricanes will cause currents with a speed of approximately one meter per second. 
The passage of hurricanes will induce upwelling of subsurface waters for a temporary period. This upwelling will cause anomalous vertical current shears. Adamec and O'Brien (1978, [1]) state that strong upwelling occurs throughout the Gulf of Guinea. Vertical and horizontal current shears are also observed. in association with major current systems. Some of the strongest current shears are in equatorial undercurrents. Equatorial undercurrents exist for this site. Avery, et al (1976, [3]), concluded that the strong current shear within five degrees latitude of the equator excludes the use of plant ships within most of that region. Strong current shears are also a problem for bottom-mounted OTEC plants. 
1. Adamec, D., and O'Brien, J.J., November 1978,. "The Seasonal Upwelling in the Gulf of Guinea Due to Remote Forcing", J. Phys. Oceanogr., 8, No. 6, 1050-1060.

2. Atwood, D.K., et al., 1976, Ocean Thermal Energy Conversion, Resource Assessment and Environment Impact for Proposed Puerto Rico Site, University of Puerto Rico.

3. Avery, W.H., et al., 1976, Maritime and Construction Aspects of Ocean Thermal Energy Conversion (OTEC) Plant Ships, The John Hopkins University Applied Physics Laboratory, Laurel, Maryland.

4. Bathen, K.H., et al., 1977, "Consolidated Oceanographic and Meteorological Data for Four North Pacific OTEC Sites", University of Hawaii.

5. Bigelow; H.B., and W.T. Emundson, 1947, Wind Waves at Sea, U.S. Hydrographic Office, Washington, D.C.

6. Colburn, J.G., 1974, "The Thermal Structure of the Indian Ocean", International Indian Ocean Expedition Oceanographic Monograph No.; 2; The University Press of Hawaii; Honolulu, Hawaii.

7. Crutcher, H.I. and R.G. Quayle, 1974, "Mariners Worldwide Climatic Guide to lropical Storms at Sea", NAVAIR 501C-61, Naval Weather Service; Asheville, N.C.

8. Defense Mapping Agency Hydrographic/Topographic Center, 1978, Pilot Chart of the North Pacific, DMA Stock Numbers PILOT 557801, 557804, 557810, Washington, D.C.

9. Defense Mapping Agency Hydrographic/Topographic Center, 1978, Pilot Chart of the North Atlantic Ocean, DMA Stock Numbers PILOT 167801, 167804, 167807, 167810, Washington, D.C.

10. Defense Mapping Agency Hydrographic Center, South Pacific Ocean, Sheet IV, Pub. N.O. 623, revised 1974, Washington, D.C.

11. Defense Mapping Agency Hydrographic Center, Indian Ocean, Northern Part, Pub No. 721, 1974, Washington, D.C.

12. Defense Mapping Agency Hydrographic Center, Gulf of Mexico and Carribean Sea, Pub. No. 410, revised 1973, Washington, D.C. 
13. Defense Mapping Agency Hydrographic Center, North Atlantic Ocean, Southeastern Sheet, Publication N.O. 125, 4th ed., 1974, Washington, D.C.

14. Düing, W., 1970; "The Monsoon Regime of the Currents in the Indian Ocean", International Indian Ocean Expedition Oceanographic Monograph No. 1, Hawaii Institute of Geophysics Contribution No. 331, the University of Hawaii Press; Honolulu, Hawaii.

15. Düing, W. and F. Schott; March 1978, "Measurements in the Source Region of the Somali Current during the Monsoon Reversal", J. Phys. Oceanogr., 8, 278-289.

16. Emilsson, J., 1970, "On the Upper Layer Circulation in the Cayman Sea", from the Symposium on the Investigations and Resources of the Caribbean Sea and Adjacent Regions, UNESCO, Paris France.

17. Fleet Numerical weather Central (FNWC), FNWC and National Oceanographic Data Center digitized reports.

18. Gentry, R.C., 1970, "Hurricanes, One of the Major Features of Air-Sea Interaction in the Caribbean sea", from the Symposium on the Investigation and Resources of the Caribbean Sea and Adjacent Regions, UNESCO, Paris, France.

19. Hogben, N., and F. Lumb, 1967, Ocean Wave Statistics, National Physical Laboratory, Ministry of Technology, London. Her Majesty's Stationery office.

20. Johnson, A. and S. Denwick, 1978; "Data Report Buoy Observations During Hurricanes Anita and Babe, AugustSeptember, 1977", NOAA Data Buoy Office, National Space Technology Laboratories, NSTL Station; Mississippi.

21. Leipper, D.F., 1967, "Observed Ocean Conditions and Hurricane Hilda, 1964. J. Atmos Sci., 24, p 182-196.

22. McFadden J.D., "Airborne Investigations of the Effects of Hurricanes on the Thermal structure of the surface Layer of the Ocean", from the Symposium on Investigation and Resources of the Caribbean Sea and Adjacent Regions, UNESCO, Paris France.

23. National Oceanographic Data Center, The Variability of Water Masses in the Indian Ocean, Publication G-11, Washington, D.C. 
24. National Oceanographic Data Center, Data File BTG75A, Washington, D.C., 1977.

25. National Oceanographic Data Center, Data Files SD40A5/0A6 from SD76A (1/30/76) 3509 STA: Washington, D.C., 1977.

26. National Science Foundation; 1972, Meteorological Atlas of the International Indian Ocean Expedition, Volume 7, Washington, D.C.

27. O'Brien, J.J., and R.O. Reid, 1967, "The Non-Linear Response of a Two Layer Baroclinic Ocean to a Stationery Axially Symetric Hurricane", J. Atmos. Sci., 24, p 197215 .

28. Ramage, C.D., 1972, "Indian Ocean Surface Meteorology", International Indian Ocean Expedition, Collective Reprints VIII, Contribution No. 624, pgs 407-540; Paris, France.

29. Sirvastava, P.S., P.K. Vyayarayou and M.X. Joseph, 1972 "Monthly Wave Characteristics of the Bay of Bengal", International Indian Ocean Expedition, Collective Reprints VIII, Contribution No. 625, Paris, France.

30. The Enclyopedia of Oceanography, 1966, Reinhold Publishing Corp., New York, New York.

31. U.S. Department of Agriculture, Weather Bureau, 1938, "Atlas of Climatic Charts of the Oceans", Washington, D.C:

32. U.S. Department of Commerce, NOAA, 1971, Eastropac Atlas, Volume 3, Washington, D.C.

33. U.S. Navy, 1975, Marine Climatic Atlas of the World, Volume III, Indian Ocean, Washington, D.C.

34. U.S. Navy Hydrographic Office, 1964, Atlas of Sea and Swell Charts, Northeastern Pacific Ocean, Publication No. 799D, revised 1976; Washington, D.C.

35. U.S. Naval Weather Service, September 1974, Climatic Summaries For Major Indian Ocean Ports and Waters, NAVAIR 50-IC-63, Asheville, N.C.

36. U.S. Naval Weather Service, November 1973, Climatic Summaries For Major Seventh Fleet Ports and Waters, NAVAIR 50-1C-62, Asheville, N.C.

37. U.S. Naval Oceanographic Office, 1963, Oceanographic Atlas of the North Atlantic Ocean, Washington, D.C. 
38. U.S. Naval Oceanographic Office, Bathymetric Atlas of the Northeastern Pacific Ocean, Pub No. 1303-S, No. $0902 \mathrm{~N}, 0903 \mathrm{~N}, 1002 \mathrm{~N}, 1003 \mathrm{~N}$, Washington, D.C.

39. U.S. Naval Oceanographic Office, Indian Ocean, AfricaEast Coast Publication No. 724, lst ed., 1974, Washington, D.C.

40. U.S. Naval Oceanographic Office, 1971, Eathymetric Atlas of the Northeastern Pacific Ocean, H.O. Pub No. $1303-\mathrm{S}$, N.0. $1104 \mathrm{~N}, 1105 \mathrm{~N}, 1204 \mathrm{~N}, 1204 \mathrm{~N}$, Washington, D.C.

41. U.S. Naval Oceanographic Office, 1971, Bathymetric Atlas of the Northeastern Pacific Ocean, H.O. Pub N.O. $1301-S, 2403 \mathrm{~N}, 2040 \mathrm{~N}$, Washington, D.C.

42. U.3. Naval Oceanographic Office, 1970, Bathymetric Atlas of thr Northweslusil. Pulfic Ueean, H.O. Pub. 1201-S, N.O. 2203N, washington, D.C.

43. Wolff, P.M. and W.E. Hubert, 1976, "Ocean Thermal Energy Conversion: Resource, Ecological and Environmental Studies", Contract No. NSF-C1020, Ocean Data Systems, Inc., Monterey, California.

44. Wolff, P.M., et al, 1977, "OTEC Resource Report for Hawaii", Contract EG-77-C-01-4028, Ocean Data Systems, Inc., Monterey, California.

45. Wyrtki, K., 1972, "The Upwelling in the Region Between Java and Australia during the South-East Monsoon, Collected reprints of the International Indian Ocean Expedition, Volume I, Contribution No. 15, pp 151-161.

46. Wyrtki, K., 1972, "Geopotential Topographics and Aseociated Circulation in the South Eastern Indian ocean", Collected reprints of the International Indian Ocean Expedition, Volume I, contribution No. 14 .

47. Wyrtki, K., 1971, Oceanographic Atlas of the International Ocean Expedition, National Science Foundation, NSF-IOE1, Washington, D.C. 